\title{
Direct photon production in high-energy nuclear collisions
}

T. Peitzmann

Citation: 1701, 060016 (2016); doi: 10.1063/1.4938679

View online: http://dx.doi.org/10.1063/1.4938679

View Table of Contents: http://aip.scitation.org/toc/apc/1701/1

Published by the American Institute of Physics 


\title{
Direct Photon Production in High-Energy Nuclear Collisions
}

\author{
T. Peitzmann \\ Utrecht University and Nikhef, Utrecht, The Netherlands
}

\begin{abstract}
Direct photons have always been considered a promising probe for the very early phases of high-energy nuclear collisions. Prompt photons reveal information about the initial state and its possible modifications in nuclei. In this context they should be one of the best probes for effects of gluon saturation. Thermal photons emitted from the produced matter in nuclear collisions carry information on the temperature of the very early phase. In particular a simultaneous measurement of yield and elliptic flow of thermal photons can put strong constraints on the early time dynamics of the system. I review the status of results on direct photon measurements at RHIC and LHC and their interpretation. Prompt photons at high $p_{T}$ are consistent with expectations from NLO pQCD in pp and show no strong nuclear modifications in A-A collisions. Recent analysis at RHIC has shown very intriguing results for lower $p_{T}$, with high thermal photon yield and strong elliptic flow of direct photons, which are not fully understood theoretically. Also the ALICE experiment at the LHC has measured a high yield of thermal photons. Furthermore I discuss prospects for future measurements of forward direct photons at the LHC.
\end{abstract}

Keywords: direct photons, relativistic heavy-ion collisions

PACS: $25.75 . \mathrm{Cj}$

\section{INTRODUCTION}

In high-energy nuclear collisions, photons are produced during all phases. Prompt photons are produced in the first (hard) scatterings of the incoming partons, so they reflect properties of the initial state. Thermal photons are generated from the thermal system throughout its entire evolution including the very early, hottest phase. As such thermal photons do contain information on the early temperature and can thus constrain properties of a possible quark-gluon plasma formed in the collisions. Moreover, as electromagnetic probes these direct photons (prompt or thermal) once produced do not interact strongly with the created system in the later phases of the collision, unlike all experimental observables based on hadrons.

Photons have the additional advantage that the elementary production processes are very well under control. Prompt photons have been studied extensively, and their measurements can be compared to precise calculations in pQCD to check the theory and also to provide input on the parton distribution functions. They can also be used to check assumptions on the dependence of particle production with centrality or system size in nuclear collisions. There has also been a lot of progress recently in the description of thermal photon production. The production rates from a quark-gluon plasma phase are very well understood, and also the calculation of rates from the hadronic phase are by now very advanced, although the latter calculations have additional uncertainties due to the fact that one has to use effective theories.

Their electromagnetic nature is also at the root of a major difficulty in direct photon measurements. The cross section for direct photon production is much smaller than that of competing processes, which also lead to photons in the final state, but which are mediated by the strong interaction. The major source of such background are neutral mesons, in particular neutral pions, which are produced in strong interaction processes and have a significant photon decay. This strong contribution of decay photons to the yield of inclusive photons leads to a small signal/background ratio for direct photon measurements, which makes this measurement very challenging. For reviews on direct photon production see e.g. $[1,2]$.

\section{PROMPT PHOTONS}

A number of different prompt photon measurements in pp collisions have been performed over a wide range of energies, and the production can in general be well described by pQCD at next-to-leading order (NLO). As an example Fig. 1 shows on the left side the cross section for direct photon production in pp collisions at $200 \mathrm{GeV}$ [3]. pQCD calculations at NLO are compared to the data - as customary three different choices for the scale parameters are 

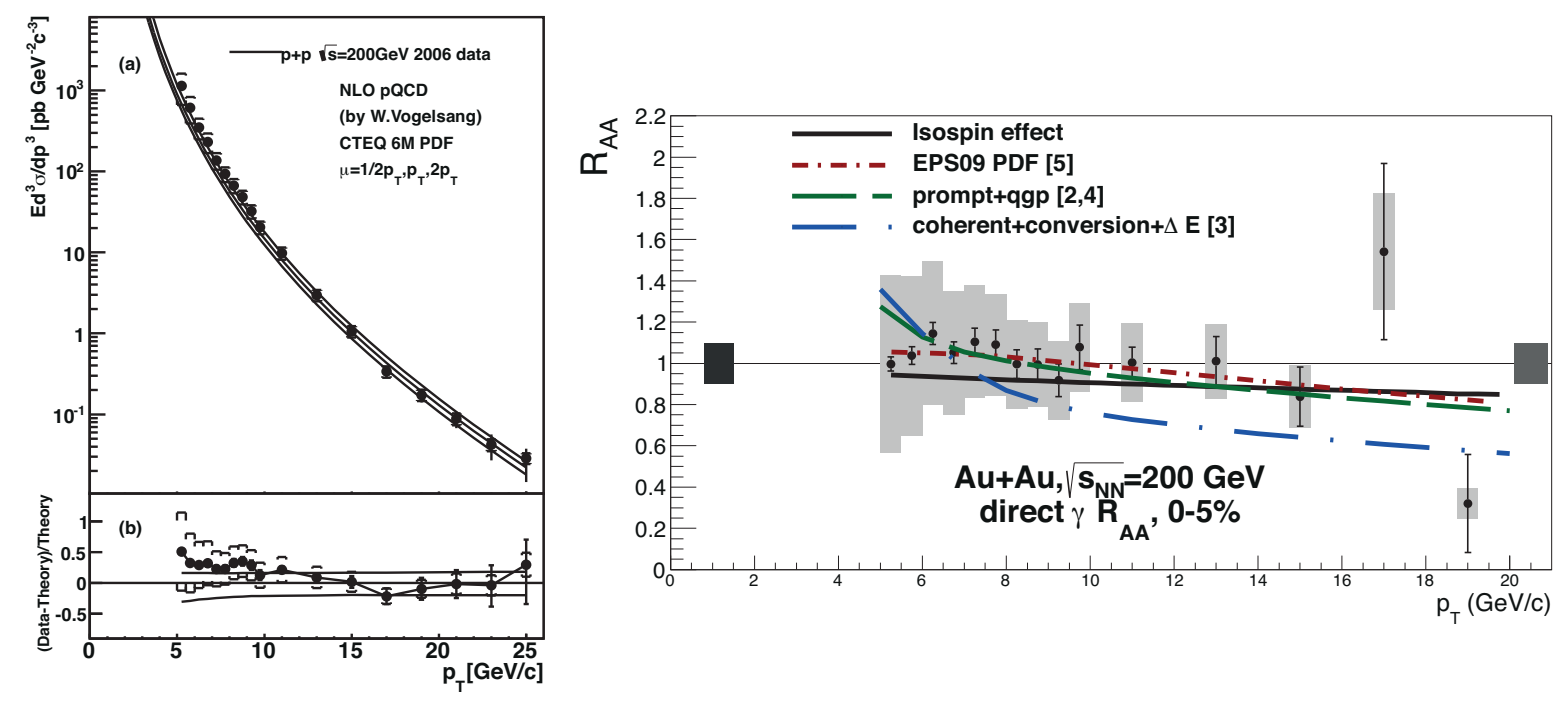

FIGURE 1. left: Cross section of direct photons in pp collisions at $200 \mathrm{GeV}$ as a function of $p_{T}$ as measured by PHENIX [3] compared to NLO pQCD calculations. right: Nuclear modification factor of direct photons as a function of $p_{T}$ for the $5 \%$ most central $\mathrm{Au}-\mathrm{Au}$ collisions at $\sqrt{s_{\mathrm{NN}}}=200 \mathrm{GeV}$ measured by PHENIX [4]. Also shown are the predictions of several theoretical calculations as discussed in the text.

used to estimate the theoretical uncertainty. The theoretical calculation agrees well with the measured data. Although there is some tension between data and theory at lower transverse momentum, the deviation is within the estimated uncertainties. The direct photon production is consistent with the expectation from prompt photons in $\mathrm{pp}$, and it is therefore useful to utilise high $p_{T}$ direct photons in nuclear collisions to establish a reference for the centrality or system size dependence of hard probes. Such dependence is usually studied via the nuclear modification factor:

$$
R_{A A}=\frac{d N / d p_{T}^{2}(A A)}{\left\langle N_{\mathrm{coll}}\right\rangle d N / d p_{T}^{2}(p p)},
$$

i.e. the ratio of the yield in $\mathrm{A}-\mathrm{A}$ collisions to the yield in pp collisions scaled with the appropriate number of nucleonnucleon collisions expected in the nuclear collision. Such a nuclear modification factor of direct photons for central $\mathrm{Au}-\mathrm{Au}$ collisions at RHIC is shown in Fig. 1 on the right. $R_{A A}$ is very close to one in this case, which is in line with the expectation of a scaling with the number of collisions for an electromagnetic hard probe such as photons. Prompt photon production is apparently not very significantly modified due to the existence of the nuclear medium. Small modifications are expected: On the one hand the different isospin, and therefore different average charge of the scattering partners in nuclei compared to protons becomes important, when one probes the valence quark content at large $p_{T}$, implying large Bjorken- $x$. This isospin effect leads to an apparent reduction of $R_{A A}$ at high $p_{T}$, as observed. Shadowing and anti-shadowing, which are included in the line labeled "EPS09 PDF", have apparently only very little effect. On the other hand, there are potential medium effects, like reduced fragmentation photon contributions due to parton energy loss, or medium induced electromagnetic radiation. Such effects are included in two other model calculations included in the figure. Both calculations show a nuclear enhancement at the low $p_{T}$ end of the spectrum, which is compatible with data due to the large uncertainties. This may be relevant for the extrapolation of the prompt spectrum down to still lower $p_{T}$ as discussed below. One of those calculations shows a significantly different result also at high $p_{T}$ - this is however not compatible with the experimental data, so one can conclude that in-medium modifications of the photon yield at high $p_{T}$ are small.

Similar measurements have been performed at the LHC by both CMS [6] and ATLAS [5]. These analyses were done for isolated photons, contrary to the results from PHENIX for inclusive direct photons. Fig. 2 shows on the left the spectra of isolated photons as a function of $p_{T}$ for $\mathrm{pp}$ and $\mathrm{Pb}-\mathrm{Pb}$ of different centrality - data from the two experiments show good agreement. There is also a reasonable agreement of MC calculations to experimental data. The right panel of Fig. 2 shows the nuclear modification factor for central $\mathrm{Pb}-\mathrm{Pb}$ collisions from $\mathrm{CMS}$, which is consistent with no nuclear modification in the measured transverse momentum range. The data are also in agreement with expectations 

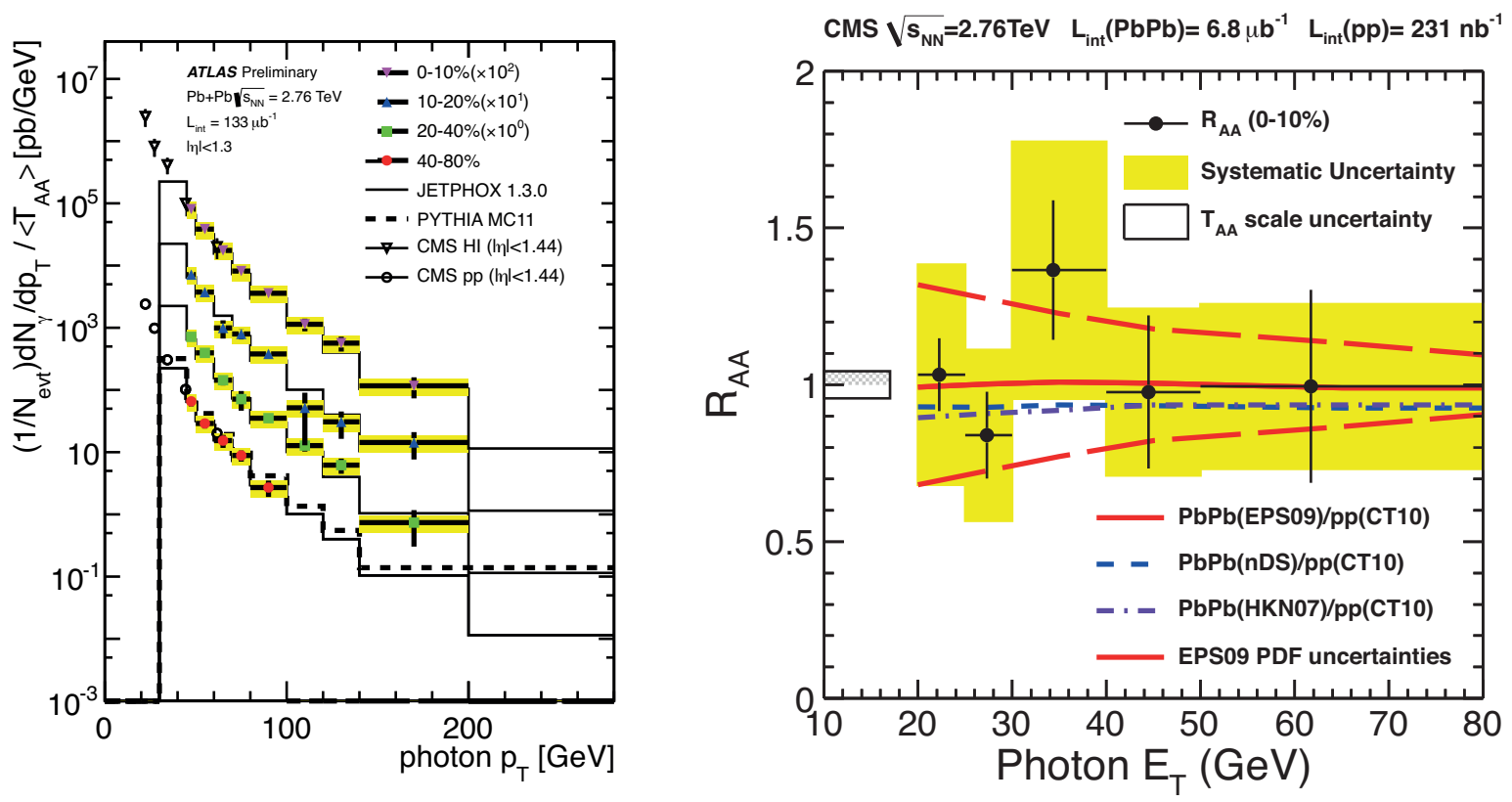

FIGURE 2. left: Yield of isolated photons in $\mathrm{Pb}-\mathrm{Pb}$ collisions of different centrality at $\sqrt{s_{\mathrm{NN}}}=2.76 \mathrm{TeV}$ normalised to the average nuclear thickness as a function of $p_{T}$ as measured by ATLAS [5] and CMS [6]. right: Nuclear modification factor of isolated photons as a function of $p_{T}$ for the $10 \%$ most central $\mathrm{Pb}-\mathrm{Pb}$ collisions at $\sqrt{s_{\mathrm{NN}}}=2.76 \mathrm{TeV}$ measured by CMS [6].

from calculations using nuclear PDFs. The isospin effect, which was visible in the RHIC data, does not show up here, because the measured $p_{T}$ values correspond to significantly lower values of Bjorken- $x$ at LHC.

The measurements of nuclear modification factors $R_{A A} \approx 1$ for direct photons at high $p_{T}$ both at RHIC and LHC demonstrate that the production of hard probes should ideally scale with the nuclear thickness (or the number of collisions), as initial state modifications appear to be small in this momentum range. This confirms the interpretation of the strong suppression of high- $p_{T}$ hadrons and jets in central nuclear collisions as being due to final-state parton energy loss. One should note that the $p_{T}$ studied both at RHIC and at LHC in these studies is still not very low, as appropriate for the study of prompt photons. This also implies that possible interesting physics at small Bjorken- $x$ is not observable here - I will return to that issue later. Similarly, the study of thermal photons, which I discuss next, has to be performed at significantly smaller $p_{T}$.

\section{THERMAL PHOTONS}

Studies of thermal photons have to deal with a very small signal to background ratio due to the important contribution of decay photons (mainly from neutral pions) to the inclusive photon spectra. This background gets more and more important at low $p_{T}$. This is usually studied by looking at the ratio of the measured inclusive photon spectrum to the one expected from hadron decays, where the latter is obtained from Monte Carlo simulations adjusted to hadron measurements:

$$
R_{\gamma} \equiv \frac{N_{\gamma}^{\mathrm{inc}}}{N_{\gamma}^{\mathrm{dec}}}=1+\frac{N_{\gamma}^{\mathrm{dir}}}{N_{\gamma}^{\mathrm{dec}}} .
$$

A deviation of $R_{\gamma}$ from a value of 1 shows the existence of a direct photon contribution. Even with additional analysis cuts for background rejection the signal will be small, with the photon excess being typically of the order of $10 \%$ or less. While in general measurements in central heavy-ion collisions are more difficult compared to pp or peripheral collisions, there are effects in central events working in favour of thermal photons. Most importantly, for thermal radiation one expects a higher yield per volume for higher temperature of the system, which one expects for central collisions - in fact, in pp collisions or peripheral heavy-ion collisions one would expect no thermal contribution at all. 
In addition, the observed parton energy loss, or jet quenching leads to a reduction of the number of neutral pions and consequently of decay photons in the relevant $p_{T}$ range. In central $\mathrm{Au}-\mathrm{Au}$ collisions at RHIC as measured by PHENIX this leads to a value of $R_{\gamma} \approx 1.2$, i.e. a photon excess of approximately $20 \%$.

PHENIX has recently performed a new analysis of direct photon production in Au-Au [7].The results for the minimum-bias distribution of direct photons are shown in Fig. 3 on the left together with those from pp collisions, which include more data points at low $p_{T}$ from a zero-mass virtual photon analysis. The new $\mathrm{Au}-\mathrm{Au}$ analysis is using a $\pi^{0}$ tagging method, which provides results of higher accuracy in the $p_{T}$ range of interest for thermal photon production.

As discussed above, the agreement between pQCD calculations and the pp data is not perfect at lower $p_{T}$, so the authors of [7] have performed a fit of a parameterisation to the pp data, which is shown as a dashed black line in Fig. 3. The fit describes the data very well. There is, however, a large uncertainty of the extrapolation of this parameterisation towards lower $p_{T}$ - the estimate of this uncertainty is indicated as a grey band. In the following this parameterisation is scaled to the expected number of collisions in Au-Au collisions. This scaled fit is compared to the minimum bias $\mathrm{Au}-\mathrm{Au}$ spectra in the same figure (solid green line). One can see that the agreement of the parameterisation with data is very good at high $p_{T}$, where the distribution is expected to be dominated by prompt photons also in Au-Au. At low $p_{T}$ there is an excess of direct photons beyond this parameterisation, which is interpreted as evidence for a thermal contribution. Consequently, the parameterisation is used to subtract the contribution from prompt photons from the direct photon spectra in nuclear collisions to obtain an estimate of thermal photons for different centralities. It should be noted that the uncertainty of the extrapolation and the scaling of it to nuclear collisions contributes to the systematic error of the thermal photon yield. PHENIX assumes a large uncertainty in the $p_{T}$ range, where no data exists for pp. This does not take into account an additional uncertainty due a possible nuclear modification of the prompt photon yield, while theoretical calculations predict significant enhancement at the low end $\left(p_{T}>5 \mathrm{GeV} / c\right)$ for central $\mathrm{Au}-\mathrm{Au}$ collisions as seen in the right panel of Fig. 1. Here more theoretical input on the extrapolation and scaling of the prompt photon yield would be very helpful.

The transverse momentum spectra of direct photons after the subtraction of the estimate of the prompt contribution obtained from scaling the pp parameterisation are shown on the right side of Fig. 3. For all centralities there is a significant direct photon excess, which may be attributed to thermal photons. The shape of the subtracted spectra is, however, very similar in all cases. They can be well described with exponential fits, which are also shown in the figure. All of these fits yield very similar inverse slope parameters of the order of $T \approx 240 \mathrm{MeV}$. Such slope parameters may be interpreted as a convolution of an effective temperature and a flow velocity of the photon source. It is therefore remarkable that these show no centrality dependence, as one would naively expect a higher effective temperature or a higher flow velocity (or both) for more central collisions. The yield depends strongly on centrality - the authors of [7] have studied the dependence on the number of participants and extract a power law with an average exponent of $\alpha=1.48 \pm 0.08$ (stat) \pm 0.04 (syst), which is expected qualitatively.

The PHENIX experiment has also been able to measure the azimuthal anisotropy of direct photon production. This is a challenging analysis, because on has to extract the anisotropy in the presence of a large background, for which a precise estimate of its own anisotropy has to be obtained, and then its effect on the inclusive photon anisotropy has to be subtracted. A recent analysis has confirmed earlier results on the second order Fourier coefficient $v_{2}$ and provided smaller uncertainties. The corresponding results are shown in the upper panels of Fig. 4. All three centrality bins show a significant $v_{2}$, and the strength increases for more peripheral collisions. In addition, a significant third order coefficient $v_{3}$ is observed in semi-central collisions (lower middle panel of Fig. 4), while the results for other centralities (left and right) are consistent with a $v_{3}$ of the same magnitude.

No theoretical estimates of $v_{3}$ of direct photons exists, but calculations have been performed for $v_{2}$, which has been considered a sensitive probe for the equilibration time of the hot and dense system [9]. In fact, an anisotropy of direct photons is expected to be created relatively late, rather more from the hadron gas phase than from the earliest, hot quark-gluon plasma phase. As such, for earlier equilibration one expects a higher direct photon yield due to contributions from the high temperature phase but at the same time a smaller elliptic flow, as the anisotropy is small in this early phase, and vice-versa for a later equilibration time.

Compared to model predictions, the photon yield is relatively high for the PHENIX measurements calling for early equilibration, while the large $v_{2}$ rather requires late equilibration, an apparent inconsistency, which has been called the "thermal photon puzzle". The most recent attempt at simultaneous description of yield and anisotropy in [10] can illustrate the state of the art - in these calculations the system emits most of the photons relatively close to the phase transition, which is apparently necessary to get anywhere close in the prediction for elliptic flow. They also have higher hadron gas contributions to photon emission than earlier calculations. A comparison of the calculations with PHENIX data is shown in Fig. 5. The left panel shows the calculated $p_{T}$ spectra together with an earlier version of the PHENIX 

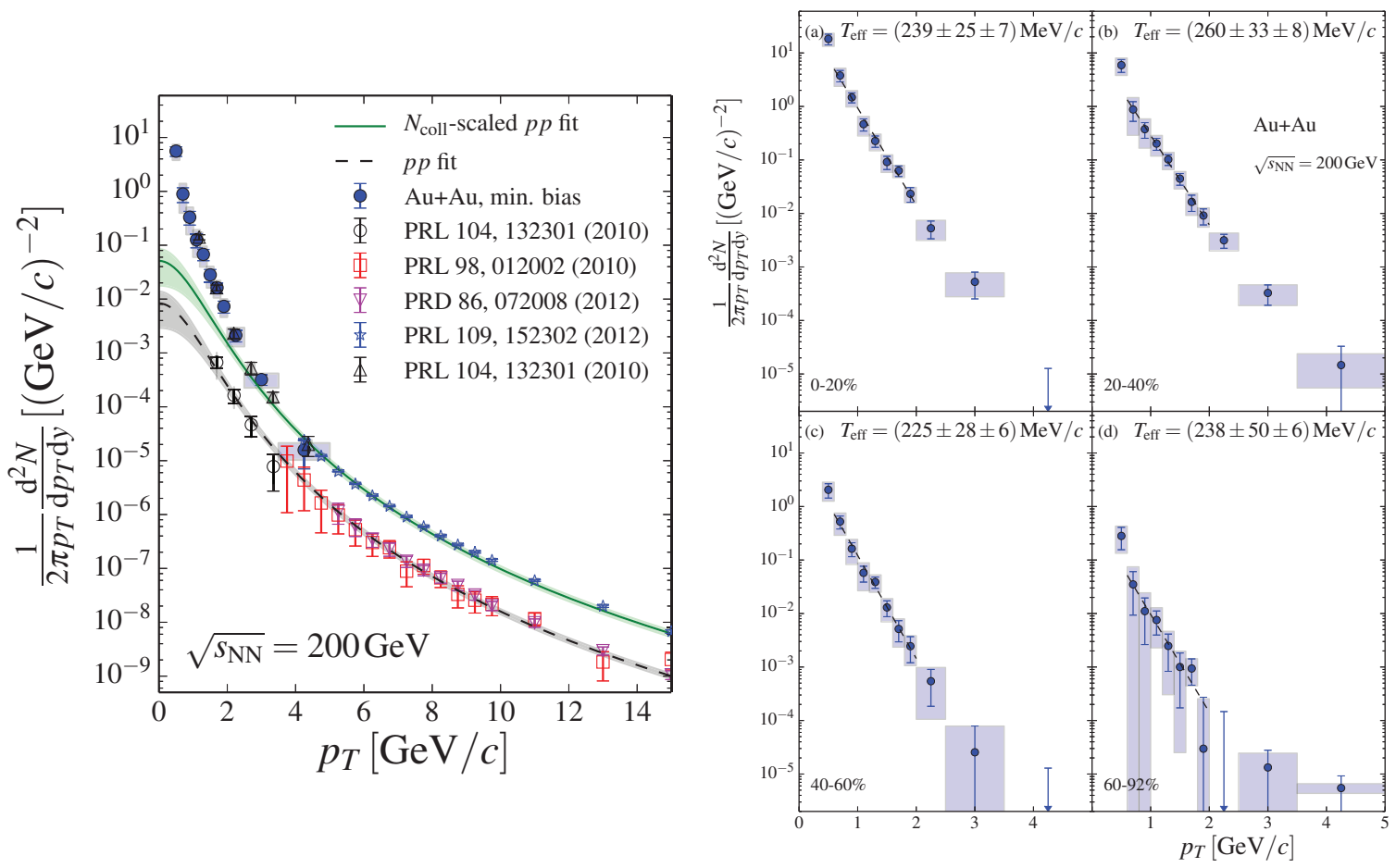

FIGURE 3. left: Yield of direct photons in pp and minimum bias Au-Au collisions at $\sqrt{s_{\mathrm{NN}}}=200 \mathrm{GeV}$ as a function of $p_{T}$ as measured by PHENIX [7]. The dashed black line shows a fit to the pp data, the solid green line the same fit scaled with the number of collisions in $\mathrm{Au}-\mathrm{Au}$ collisions. right: Direct photon $p_{T}$ spectra after subtraction of the $N_{\text {coll }}$-scaled pp parameterisation for $\mathrm{Au}-\mathrm{Au}$ collisions of different centrality. The dashed lines are fits of an exponential function.

data, which is fully compatible with the new results. The default calculation is not able to describe the data, the yield is too low in particular at low $p_{T}$. When the rate of emission is enhanced significantly (by a factor of 3 in the vicinity of the phase transition, which the authors themselves call "a pseudo-critical enhancement"), the calculated yield is much closer to the data, although it does still not fully explain the yield. In the right panel of Fig. 5 a similar comparison is performed for the elliptic flow strength. Again the default calculation falls short of the data, while the agreement is better using the enhanced rates. This is certainly a very important observation - however, it remains to be demonstrated that the rate enhancement can be motivated from microscopic calculations, as the authors themselves also admit.

First results from direct photon measurements are also available from the ALICE experiment at the LHC. One expects a higher thermal photon yield, but due to the flatter spectra of hadrons, e.g. neutral pions, which contribute to the decay photon spectrum, the signal-to-background ratio is worse for the LHC compared to RHIC, making the measurement even more challenging. The resulting direct photon spectrum for central $(0-40 \%) \mathrm{Pb}-\mathrm{Pb}$ collisions at $\sqrt{s_{\mathrm{NN}}}=2.76 \mathrm{TeV}$ is shown in Fig. 6 [11]. The figure also includes results of a pQCD calculation, which agree very well with the experimental data at high $p_{T}$, indicating that that $p_{T}$ rage is dominated by prompt photons. However, as at RHIC, there is an excess of direct photons beyond the expectation from the prompt yield at low $p_{T}$. Two recent hydrodynamical calculations, which are shown for comparison, predict a significantly smaller yield. The calculation of [10] (not shown here) can describe the measurements reasonably well. The low- $p_{T}$ part of the spectrum can also be described with a simple exponential fit, which yields an inverse slope parameter of $T \approx 300 \mathrm{MeV}$, somewhat higher than at RHIC, as expected.

ALICE is also performing an analysis of direct photon elliptic flow. As this depends very critically not only on the magnitude of errors on the direct photon yield, but also on the possible correlation of errors as a function of $p_{T}$, the results await a reevaluation of systematic errors. 


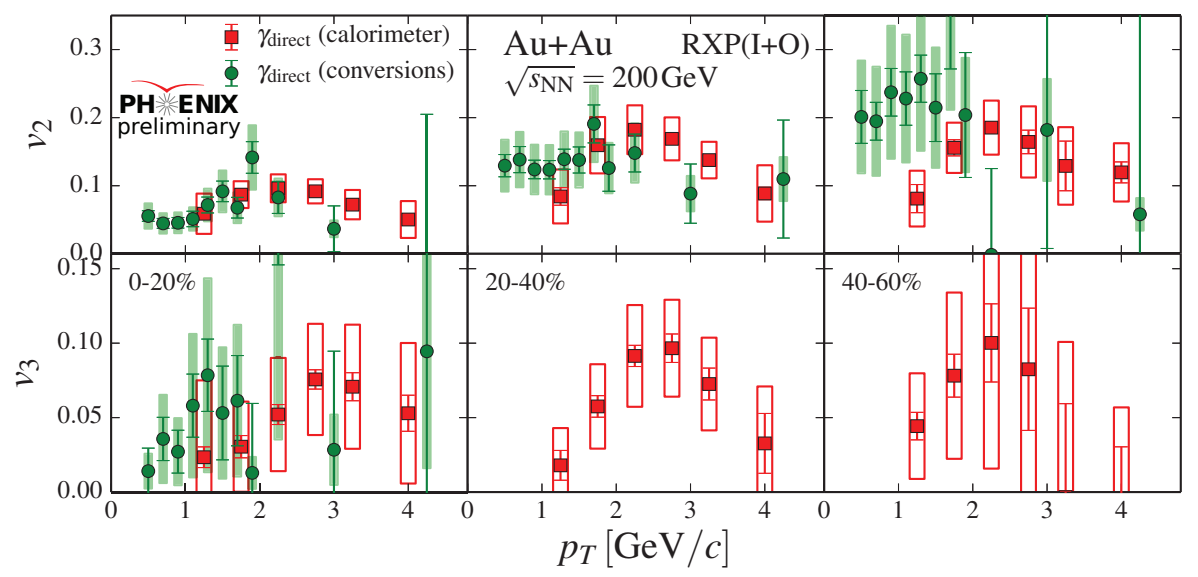

FIGURE 4. Fourier coefficients $v_{2}$ and $v_{3}$ of the azimuthal anisotropy of direct photons for $\mathrm{Au}-\mathrm{Au}$ collisions of different centrality at $\sqrt{s_{\mathrm{NN}}}=200 \mathrm{GeV}$ as a function of $p_{T}$ as measured by PHENIX [8].

\section{PHOTONS AS SIGNAL FOR GLUON SATURATION}

As mentioned above, direct photons can be used as a probe of the parton distribution in protons and nuclei. The gluon PDF is not very well constrained, even more so for nuclei, and in particular at low values of Bjorken- $x$. Here DGLAP evolution predicts a dramatic increase of the gluon density, which would lead to fundamental problems, e.g. with unitarity. It is expected that non-linear effects (gluon fusion or recombination) would limit the growth and would lead to gluon saturation $[12,13,14]$.

Gluon saturation should effect the total multiplicity of produced particles in high-energy collisions. Furthermore, the predicted scaling properties of the momentum scales should lead to a behaviour called geometric scaling. Both the multiplicities and the scaling properties of particle distributions have been measured and compared to saturation models. The results appear to be consistent with expectations from the models, but unfortunately these observables are not specific enough to provide a proof for gluon saturation.

More discriminatory power may come from more detailed studies of transverse momentum distributions and from two-particle angular correlations. The main interest lies here in the comparison of particle production in $\mathrm{pp}$ and $\mathrm{p}-\mathrm{A}$
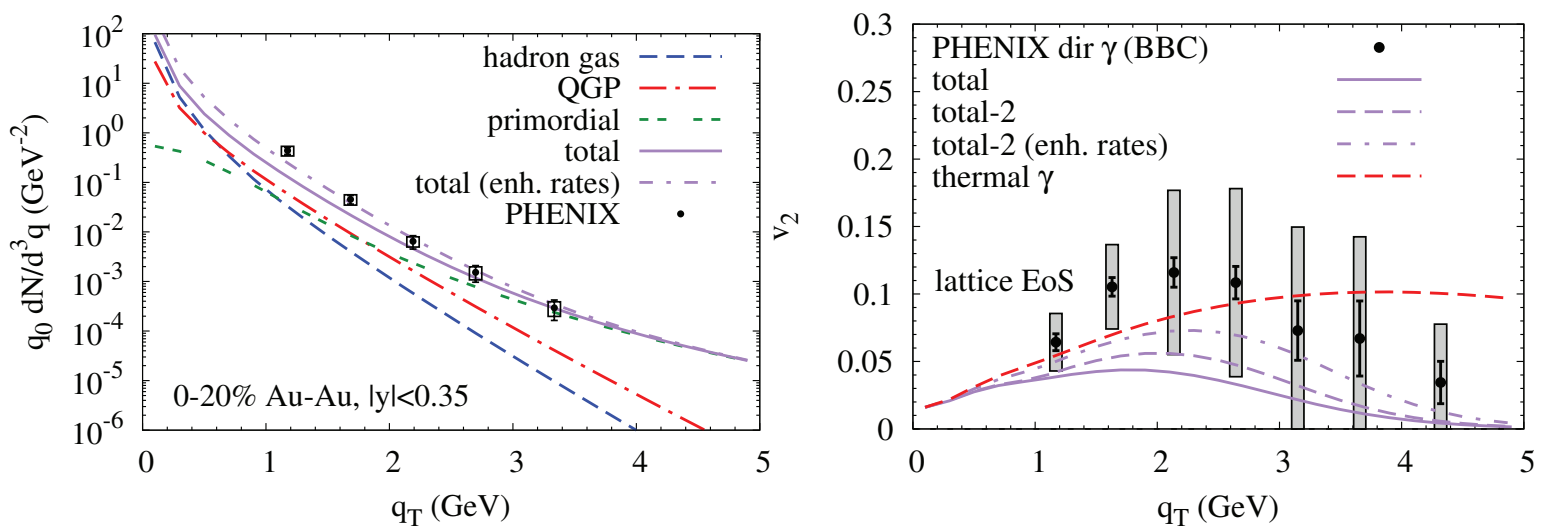

FIGURE 5. Comparison of theoretical calculations to the direct photon measurements of PHENIX (from [10]). The figures also include the results of the calculation for an arbitrarily enhanced rate. left: Transverse momentum spectra. The different components included in the calculations are shown separately. right: Elliptic flow strength as a function of $p_{T}$. 
collisions, as saturation effects should be stronger for the higher gluon density in nuclei. Specifically, one expects that

- the nuclear modification factor in p-A collisions should show a suppression of particle production $R_{p A}<1$ in a characteristic $p_{T}$ range, and

- the jet-like peak at $\Delta \phi=0$ usually observed in two-particle correlations in pp collisions should be modified (weakened and/or broadened) in nuclear collisions.

The interesting kinematic range is defined by the values of Bjorken $x$ and the corresponding saturation scale $Q_{s}$. The relevant production processes will be affected by saturation when $Q<Q_{s}$, which calls for small to intermediate momentum probes. However, as one would like to use a calculable probe as a reference, this excludes too low momentum transfers - ideally one would want to study momentum ranges, where perturbative QCD should be applicable. To access small $x$, which for leading-order processes on the parton level can be approximated as $x \approx$ $2 p_{T} \exp (-y) / \sqrt{s}$, particle production at large (i.e. forward) rapidities should be studied.

Transverse momentum spectra and angular correlations for neutral pions at forward rapidity have been studied in pp and d-A collisions at RHIC, and a suppression has been observed in the nuclear modification factor $[15,16]$ and a suppression and broadening in the angular correlation [17, 18]. However, the transverse momenta studied are still very small (on the order of $1 \mathrm{GeV}$ or only slightly higher), a momentum range where particle production is anyhow not well understood. In addition, the relation between the kinematic variables in the final state are only weakly correlated to the parton kinematic parameters due to fragmentation and possibly other final state modifications of particle production.

Similar studies at LHC should allow to study both higher $Q^{2}$ and smaller $x$ contributions, thus should be able to use well-defined particle production processes, while still being sensitive to saturation as the saturation scale should be much larger at the lower $x$ values. First measurements of hadron production at forward rapidities have been performed at the LHC, but results are not conclusive. The production of $\phi$ mesons shows a strong suppression in $\mathrm{p}+\mathrm{A}$ collisions compared to pp [19]. There is also a suppression of $J / \psi$ production [20,21], which is consistent with calculations using shadowing and final state energy loss. One CGC calculations predicts a stronger suppression than seen in the data, however this calculation has a number of uncertainties related to the coupling of the $J / \psi$ to the gluon field, and it does not use a state-of-the-art CGC implementation. In general, also at LHC, hadron production will most likely not provide an ideal probe because of final state modifications and their uncertainties, which will obscure the kinematics.

Probes, which directly access the parton kinematics, would be strongly preferable, which points to direct photons as an ideal probe. Direct photons have a number of advantages compared to other, in particular hadronic probes:

1. The production processes of direct photons are well understood theoretically.

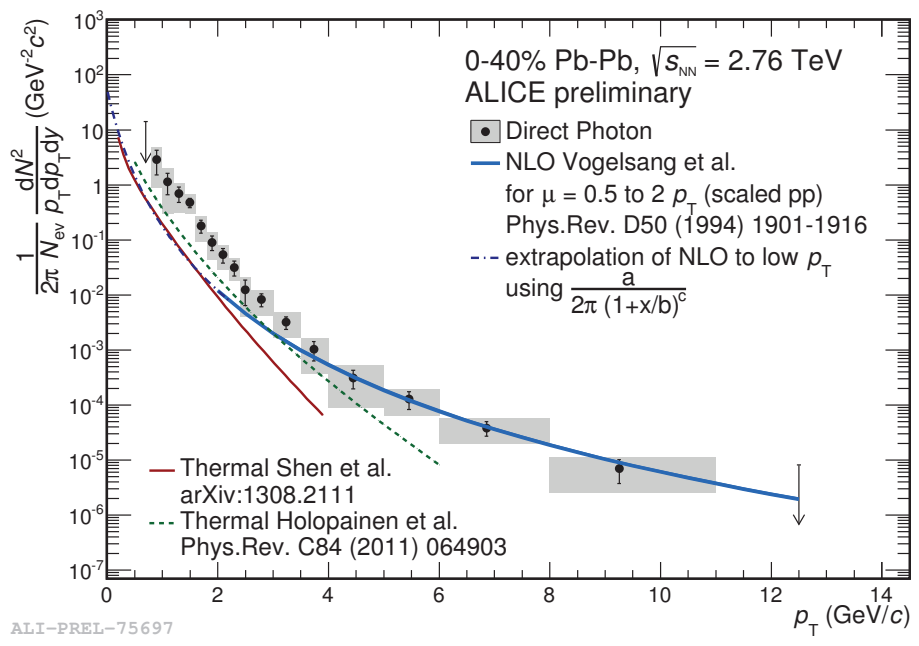

FIGURE 6. Yield of direct photons in central $\mathrm{Pb}-\mathrm{Pb}$ collisions at $\sqrt{s_{\mathrm{NN}}}=2.76 \mathrm{TeV}$ as a function of $p_{T}$ as measured by ALICE [11]. The solid blue shows a pQCD calculation at NLO for pp scaled with the number of collisions in $\mathrm{Pb}-\mathrm{Pb}$ - the results for this calculation are extrapolated towards lower $p_{T}$, which is included as a dash-dotted line. The solid red and dashed green lines show predictions of hydrodynamical calculations for the thermal photon yield. 
2. The leading order process (q-g Compton scattering) is directly sensitive to the gluon density.

3. Fragmentation contributions, though significant at LHC, are less important than for hadrons, and can be suppressed by isolation cuts.

4. No other strong final state nuclear modification (like e.g. energy loss) is expected.

The advantage of photons with respect to the sensitivity to parton kinematics can be illustrated with the distribution of momentum fractions $x_{2}$ probed in the nucleus in p+A reactions at the LHC as displayed in the left panel of Figure 7. The $x_{2}$ distribution for photon production with $4<y<5$ and $5<p_{T}<20 \mathrm{GeV} / c$ is peaked between $10^{-5}$ and $10^{-4}$, while the maximum contribution for pion production is generated from partons with $x_{2}$ about an order of magnitude larger. Thus, already the inclusive direct photon distribution has a clear sensitivity advantage, which can be further enhanced by applying isolation cuts on the photons, although the studies in Ref. [22] show that isolation may not be as effective as previously thought.

Another advantage of photon production is that the theoretical description also in the context of models of gluon saturation is very well understood. State-of-the-art calculations have shown a clear sensitivity to gluon saturation effects, as demonstrated in the right panel of Figure 7, which shows the nuclear modifications factor $R_{p A}$ of direct photons from the CGC calculation in Ref. [23] and from a pQCD calculation at NLO with EPS09 PDFs using JETPHOX. While the pQCD prediction shows only a slight reduction of reaching $R_{p A} \approx 0.8$ at low $p_{T}$ related to nuclear shadowing, the CGC calculation shows a strong suppression to $R_{p A}<0.4$ for $p_{T}<5 \mathrm{GeV} / c$.

In principle the measurement of Drell-Yan production - i.e. virtual direct photons - would provide an alternative means of assessing low-x parton distributions with similar advantages, i.e. no final state modifications. The measurement is not as directly accessing the gluon distribution, however, as gluons play a role only via second order diagrams, or in the quark/antiquark PDFs via DGLAP evolution. It may be an additional complication for the interpretation to rely heavily on DGLAP evolution in theoretical predictions - finally a search for gluon saturation effects should challenge DGLAP evolution. Still this is likely not a major argument against using Drell-Yan.

The major disadvantage of Drell-Yan measurements is the very low cross section compared to real photon production. Ref. [24] shows a measurement of forward Drell-Yan muon pairs in pp collisions at $7 \mathrm{TeV}$ from LHCb. For the low mass range relevant for this discussion $(5<M<7 \mathrm{GeV})$ the authors quote a statistical error of $\approx 20 \%$ for the rapidity-integrated measurement in a sample of $37 \mathrm{pb}^{-1}$. A low-mass rapidity-differential measurement would not be possible from this sample. The situation is considerably worse for $\mathrm{p}+\mathrm{Pb}$ collisions, where an integrated luminosity of $50 \mathrm{nb}^{-1}$ (corresponding to a nucleon-nucleon-equivalent luminosity of $\approx 10 \mathrm{pb}^{-1}$ ) is considered reasonable. Measurements of Drell-Yan production will therefore not be competitive to those of real photon production.
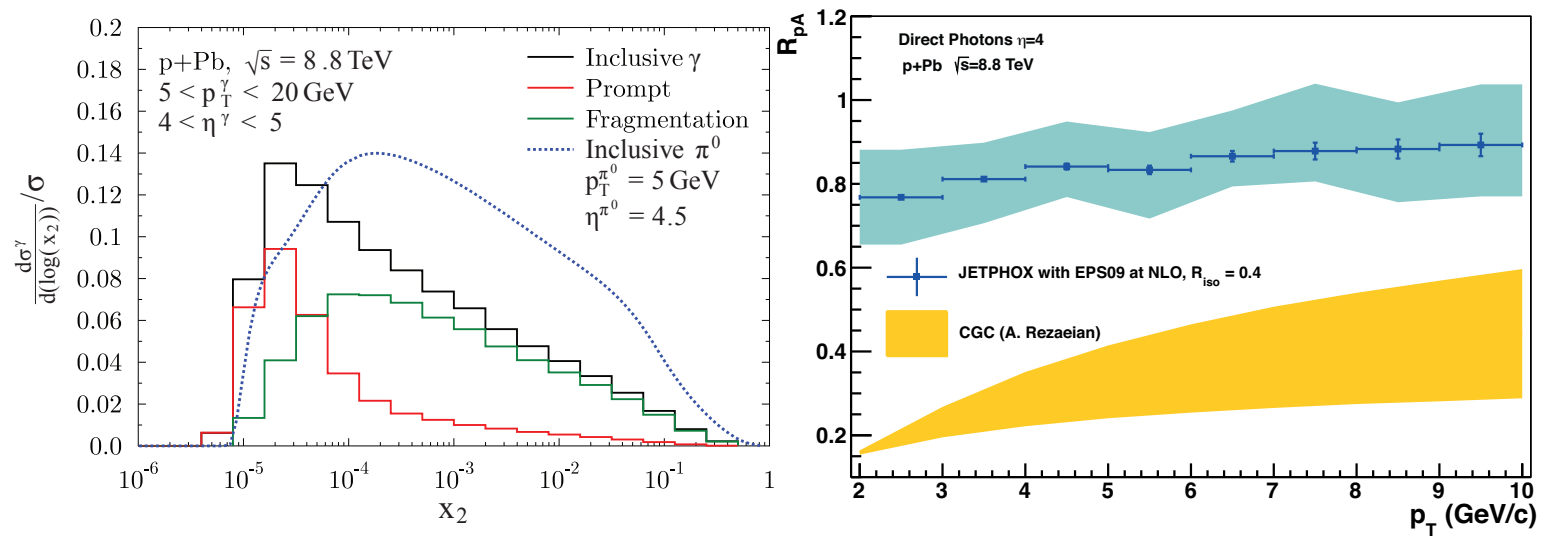

FIGURE 7. left: Distribution of $x_{2}$ (momentum fraction of parton from the nucleus) probed in direct photon production at forward rapidity in $\mathrm{p}+\mathrm{Pb}$ collisions at $8.8 \mathrm{TeV}$ as calculated in JEPHOX using EPS09 structure functions in Ref. [22]. The different components of photon production are also shown separately. For comparison, the $x_{2}$ distribution for pion production of similar kinematics are included. right: Nuclear modification factor $R_{p A}$ as a function of $p_{T}$ for forward direct photon production. Shown are results of CGC calculations from Ref. [23] (orange) and from NLO pQCD calculations with JETPHOX (blue). The shaded bands show the systematic error estimates. 

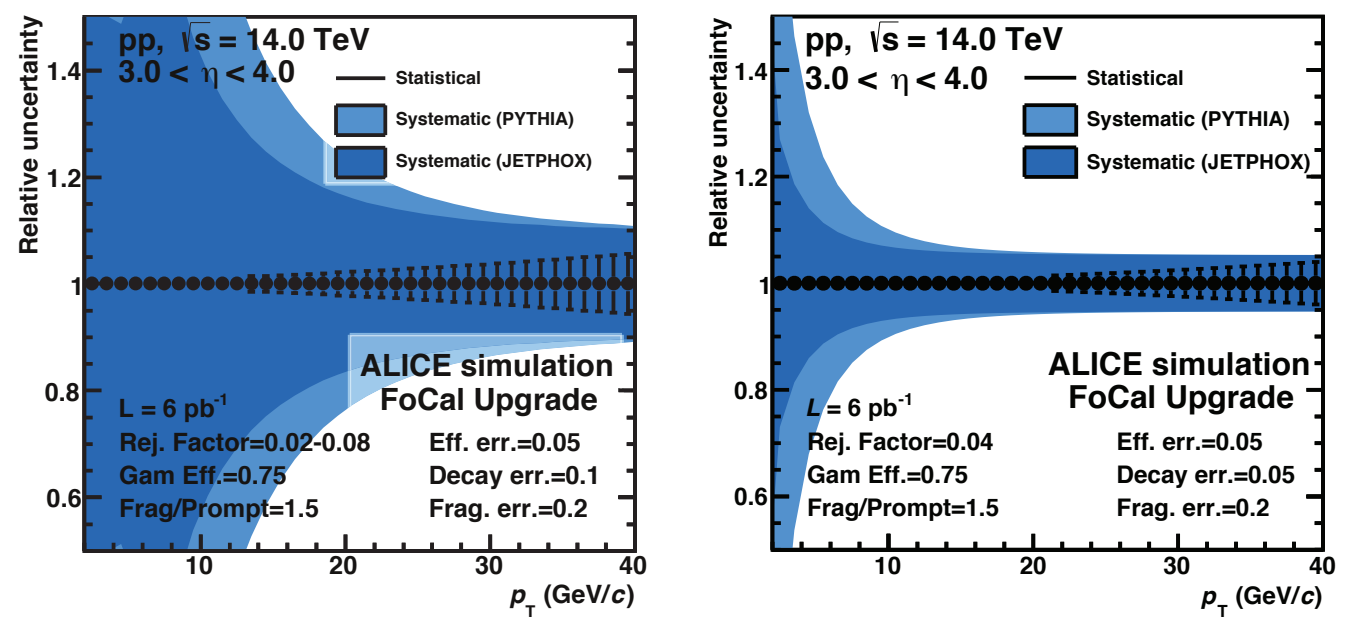

FIGURE 8. Estimated relative uncertainties on the cross section measurement for direct photon production in $\mathrm{p}+\mathrm{p}$ collisions at $\sqrt{s}=14 \mathrm{TeV}$, based on direct photon spectra from JETPHOX (dark band) and PYTHIA (light band), and background spectra from PYTHIA events. Statistical uncertainties are shown as error bars and the systematic uncertainty is shown as a band. This simulation assumes a location of the detector at $z=3.6 \mathrm{~m}$. Results are shown using only low granularity layers (left) and for the full detector including high granularity layers (right).

A new detector for measurements of forward direct photons in ALICE. A detector upgrade with a calorimeter at forward rapidities (FoCal) to measure forward direct photon production is currently being discussed in the ALICE collaboration [25]. This detector would be intended to measure direct photons, electrons/positrons and jets for rapidities $\eta>3$. Such a detector would offer a wealth of physics possibilities, but its main focus is on measurements related to the structure of nucleons and nuclei at very low Bjorken- $x$ and possible effects of gluon saturation.

FoCal would consist of an electromagnetic calorimeter most likely positioned at a distance from the IP of $z \approx 7 \mathrm{~m}$ covering $3.2<\eta<5.3$ backed by a standard hadronic calorimeter. A distance of $z=3.6 \mathrm{~m}$, which corresponds to a maximum reachable pseudorapidity of $\eta=4.5$, has also been studied in simulations. Both positions are equivalent in terms of measurement conditions such as the particle density, such that it is sufficient at this stage to not explicitly perform all studies for both positions. The main challenge of an electromagnetic calorimeter in this region of phase space is the requirement to discriminate decay photons from direct photons at very high energy, which will require extremely high granularity.

The design option currently under study is a SiW sandwich construction. It consists of 20 layers of a $3.5 \mathrm{~mm} \mathrm{~W}$ plate $\left(\approx 1 X_{0}\right)$ interleaved with active layers with $\mathrm{Si}$ sensors. The active layers use two different sensor technologies: low granularity layers (LGL), which consist of sensors with $1 \mathrm{~cm}^{2}$ pads summed longitudinally in segments and equipped with analog readout, and high granularity layers (HGL) based on CMOS monolithic active pixel sensors (MAPS). The MAPS will have a pixel size of a few $10 \times 10 \mu \mathrm{m}^{2}$ with internal binary readout. ${ }^{1}$ On-chip processing will convert the pixel count in a macro-pixel of $1 \mathrm{~mm}^{2}$ to a pseudo-analog value.

The HGL are crucial for $\gamma / \pi^{0}$ discrimination. The LGL have an effective tower width of the order of the Molière radius. Their two-shower separation power is similar to existing standard electromagnetic calorimeters. ${ }^{2}$ However, the shape of electromagnetic showers allows us to make use of much finer granularity for shower separation and additional shower shape analysis for very high energy $\pi^{0}$, when the two photons can no longer be resolved. The impact of the granularity is shown in Fig. 8, which displays uncertainty estimates for a direct photon measurement in pp collisions at $14 \mathrm{TeV}$. The panel on the left hand side shows the expected performance using only the LGL, while the right panel shows the performance of the full detector. A low-granularity detector would only determine the photon yield with a much larger systematic error, mainly due to the merging of $\pi^{0}$-decay photons. Only the high-granularity option has

\footnotetext{
1 The current model for MC simulations uses a pixel size of $100 \times 100 \mu \mathrm{m}^{2}$.

2 Those conditions are in fact very similar to the ones of the electromagnetic calorimeter of the LHCb experiment.
} 


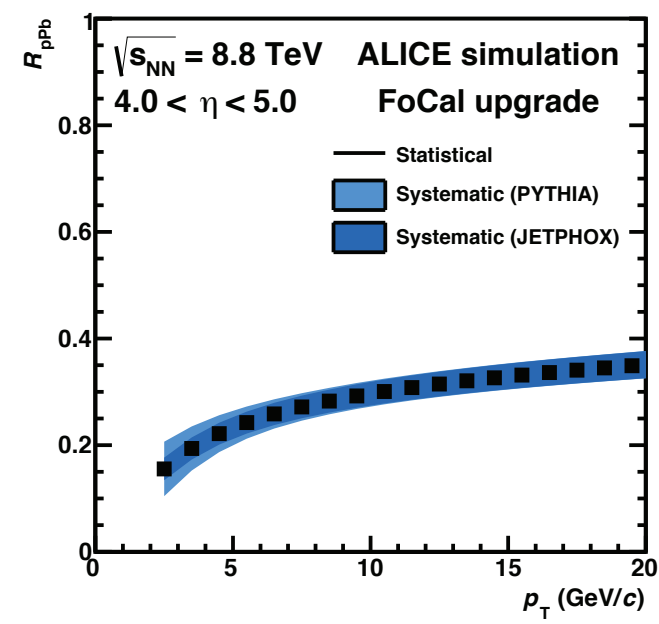

FIGURE 9. Estimated relative uncertainties on measurement of the nuclear modification factor $R_{p A}$ for direct photon production at $\sqrt{s}=8.8 \mathrm{TeV}$, based on direct photon spectra from JETPHOX (dark band) and PYTHIA (light band), and background spectra from PYTHIA events. Statistical uncertainties are shown as error bars and the systematic uncertainty is shown as a band. This simulation assumes a location of the detector at $z=3.6 \mathrm{~m}$. Results are shown using only low granularity layers (left) and for the full detector including high granularity layers (right).

a good sensitivity for such a photon measurement. While FoCal would offer coverage towards higher rapidities than other LHC experiments, it is in particular the superior granularity at these large rapidities that would give FoCal a unique advantage.

The detector upgrade would be installed in the LHC long shutdown $3(\approx 2024)$, and measurements would be performed together with the full ALICE setup. Beam intensity conditions should thus be similar to the standard requirements of the upgraded ALICE experiment.

\section{CONCLUSION}

An overview of recent results on direct photon production in nuclear collisions has been given. Prompt photons have been measured both at RHIC and LHC. The production at high $p_{T}$ is well described by NLO pQCD calculations. In particular, prompt photon production is consistent with a scaling with the number of NN-collisions in nuclear collisions, i.e. no strong nuclear modification is observed. This is an additional confirmation that the suppression of high $p_{T}$ hadrons and jets in central collisions is a final-state, strong-interaction effect and is most likely attributed to parton energy loss.

For low- $p_{T}$ direct photons an excess beyond the expectations from prompt production is observed, again both at RHIC and at LHC. The excess has an approximately exponential shape, which is consistent with an effective inverse slope of $T \approx 240 \mathrm{MeV}$ at RHIC and $T \approx 300 \mathrm{MeV}$ at LHC. The thermal photon yield increases strongly for more central collisions, and is also higher at the LHC energy. For precision comparisons of the thermal yield to theoretical calculations another investigation of uncertainties of prompt photon production at low $p_{T}$, e.g. related to possible inmedium modifications, should be performed. Also measurements of elliptic flow of direct photons has been performed at RHIC, and results are forthcoming from ALICE at the LHC. AT RHIC a surprisingly large elliptic flow is observed.

Current theoretical model calculations have difficulties to describe both the high yield of thermal photons and the large values of $v_{2}$ - apparently the only possibility for a good description lies in assuming a significantly enhanced rate of thermal photon emission, in particular close to the phase transition temperature.

Moreover, direct photons at forward rapidities at the LHC are considered a very promising probe for gluon saturation. A very strong suppression of the yield in $\mathrm{p}-\mathrm{A}$ is predicted by saturation models compared to "standard" pQCD. Such forward measurements require a photon detector going beyond state-of-the-art, in particular with respect to the two-shower separation power, requiring a much higher granularity that currently available. 
Within the ALICE experiment, a SiW calorimeter (FoCal) including layers of extremely high granularity is discussed as a possible upgrade. Such a detector would allow superior studies of effects of gluon saturation.

\section{ACKNOWLEDGMENTS \\ REFERENCES}

1. T. Peitzmann, and M.H. Thoma, Phys. Rept. 364 (2002) 175-2468.

2. P. Stankus, Ann. Rev. Nucl. Part. Science 55 (2005) 517-554.

3. A. Adare et al. (PHENIX Collaboration), Phys. Rev. D 86 (2012) 072008.

4. S. Afanasiev et al. (PHENIX Collaboration), Phys. Rev. Lett.109 (2012) 152302.

5. P. Steinberg et al. (ATLAS Collaboration), preprint arXiv:1209.4910.

6. S. Chatrchyan et al. (CMS Collaboration), Physics Letters B, 710 (2012) 256 Đ277.

7. A. Adare et al. (PHENIX Collaboration), submitted to Phys. Rev. C, preprint arXiv:1405.3940.

8. B. Bannier et al. (PHENIX Collaboration), preprint arXiv:1408.0466.

9. R. Chatterjee, D.K. Srivastava, and U. Heinz, preprint arXiv:0901.3270.

10. R. Rapp, H. van Hees, and M. He, preprint arXiv:1408.0612.

11. A. Morreale et al. (ALICE Collaboration), preprint arXiv:1409.4456.

12. L.V. Gribov, E.M. Levin, and M.G. Ryskin, Phys. Rept. 100 (1983) $1 Đ 150$.

13. A.H. Mueller and J.-W. Qiu, Nucl. Phys. B268 (1986) 427.

14. L.D. McLerran and R. Venugopalan, Phys. Rev. D49 (1994) 223-2241; L.D. McLerran and R. Venugopalan, Phys. Rev. D49 (1994) 335-3355; L.D. McLerran and R. Venugopalan, Phys. Rev. D50 (1994) 222-2233.

15. J. Adams et al. (STAR Collaboration), Phys. Rev. Lett. 97 (2006) 152302.

16. I. Arsene et al. (BRAHMS Collaboration) Phys. Rev. Lett. 93 (2004) 242303.

17. E. Braidot et al. (STAR collaboration), Proceedings of the 45th Rencontres de Moriond (2010) preprint arXiv:1005.2378.

18. A. Adare et al. (PHENIX Collaboration), Phys. Rev. Lett. 107, 172301 (2011).

19. A. Uras, ALICE Collaboration, talk at Hard Probes 2013, http://indico.tlabs.ac.za/contributionDisplay.py?contribId=112\&confId=30

20. LHCb Collaboration, JHEP 02 (2014) 072.

21. B. Abelev et al. (ALICE Collaboration), JHEP02 (2014) 073.

22. I. Helenius, K.J. Eskola, H. Paukkunen, preprint arXiv:1406.1689.

23. J. Jalilian-Marian, A. Rezaeian, Phys. Rev. D 86 (2012) 034016; A. Rezaeian, private communication.

24. LHCb collaboration, LHCb-CONF-2012-013.

25. T. Peitzmann, Proceedings of CHEF 2013, preprint arXiv:1308.2585. 